Department of Mathematics

A New Multigrid Formulation
for High Order Finite Difference
Methods on Summation-by-Parts Form

Andrea A. Ruggiu, Per Weinerfelt and Jan Nordström

LiTH-MAT-R--2017/08--SE 
Department of Mathematics

Linköping University

S-581 83 Linköping 


\title{
A New Multigrid Formulation for High Order Finite Difference Methods on Summation-by-Parts Form
}

\author{
Andrea A. Ruggiu ${ }^{\mathrm{a}, *}$, Per Weinerfelt ${ }^{\mathrm{a}, \mathrm{b}}$, Jan Nordström ${ }^{\mathrm{a}}$ \\ ${ }^{a}$ Department of Mathematics, Computational Mathematics, Linköping University, \\ SE-581 83 Linköping, Sweden \\ ${ }^{b}$ Saab Aerospace, SE-581 88 Linköping, Sweden
}

\begin{abstract}
Multigrid schemes for high order finite difference methods on summationby-parts form are studied by comparing the effect of different interpolation operators. By using the standard linear prolongation and restriction operators, the Galerkin condition leads to inaccurate coarse grid discretizations. In this paper, an alternative class of interpolation operators that bypass this issue and preserve the summation-by-parts property on each grid level is considered. Clear improvements of the convergence rate for relevant model problems are achieved.
\end{abstract}

Keywords: High order finite difference methods, summation-by-parts, multigrid, restriction and prolongation operators, convergence acceleration.

\section{Introduction}

The multigrid method is a convergence acceleration technique that improves iterative solvers using grid coarsening [1,2]. This methodology leads to improved convergence for elliptic problems in a straightforward way $[2,3]$, while partial differential equations with a dominant hyperbolic character are often handled with additional artificial dissipation $[4,5]$. Multigrid methods are used in various branches of applied mathematics and engineering, such as electromagnetics [6], magnetohydrodynamics [7] and fluid dynamics [8].

\footnotetext{
*Corresponding author

Email addresses: andrea.ruggiu@liu.se (Andrea A. Ruggiu), per.weinerfelt@saabgroup.com (Per Weinerfelt), jan.nordstrom@liu.se (Jan Nordström)
} 
Two important components in multigrid methods are the restriction and prolongation operators which transfer the information between grids. Typically these operators are based on linear interpolation procedures, regardless of the accuracy of the discretization [3]. Although this is a natural choice for low order schemes, it may be inappropriate for high order ones. In this work we make use of a general relation between prolongation and restriction operators which was originally proposed in a different context [9]. The resulting interpolation operators lead to a consistent approximation at the boundaries and guarantee that the formal order of accuracy of the original scheme is retained at the interior nodes on the coarse grids.

By applying the specific grid transfer operators to Summation-by-Parts (SBP) formulations with Simultaneous-Approximation-Terms (SATs) [10] weakly imposing the boundary conditions, we arrive at a multigrid method with provable energy stable discretizations on each grid level. Moreover, the procedure allows for the introduction of the stable and accurate artificial viscosity procedure proposed in [11]. The resulting improved convergence of the new multigrid method is exemplified for several problems.

The rest of this paper is organized as follows: in Section 2 the main features of the two-level multigrid algorithm are presented. Section 3 introduces the SBP-SAT technique for high order finite difference discretizations. Section 4 deals with the construction of multigrid algorithms using the new class of interpolation operators. In Section 5 we compare the effects of different prolongation and restriction operators on the multigrid convergence. Conclusions are drawn in Section 6.

\section{The multigrid algorithm}

Consider the following steady-state problem:

$$
\begin{aligned}
L u & =f, \text { in } \Omega, \\
H u & =g, \text { on } \partial \Omega,
\end{aligned}
$$

where $L$ is a linear differential operator, $H$ is a boundary operator, $f$ and $g$ are given functions, and $\Omega$ is the domain with boundary $\partial \Omega$. The boundary conditions are assigned in a way such that (1) is well-posed $[12,13]$.

The construction of a two-level multigrid scheme [2, 3] for solving (1) consists of the following four steps:

1. Fine-grid discretization; 


\section{Error smoothing;}

3. Coarse-grid correction;

4. Fine-grid update.

In the following sections, we will outline the main features of these four steps.

\subsection{Fine-grid discretization}

Consider a grid $\Omega_{1}$, here called the fine grid, on $\Omega$. A discrete problem associated to (1) on the fine grid $\Omega_{1}$ has the general form

$$
L_{1} \mathbf{u}=\mathbf{F},
$$

where $L_{1}$ is a discrete version of the operator $L$ in (1) which also includes the boundary conditions. The vector $\mathbf{F}$ is a grid function which approximates $f$ on the nodes of $\Omega_{1}$, augmented with boundary data $\mathbf{g}$, and $\mathbf{u}$ is an approximate solution to (1). Typically $\Omega_{1}$ has many nodes, and it is expensive to solve (2) directly. Furthermore, the discrete operator $L_{1}$ is assumed to be invertible and have eigenvalues with strictly positive real parts.

\subsection{Error smoothing}

An error smoothing procedure is required prior to grid coarsening. First, we consider marching towards the solution to (2) in dual time from an initial guess $\mathbf{u}^{(0)}$ by solving

$$
\begin{aligned}
\mathbf{w}_{\tau}+L_{1} \mathbf{w}(\tau) & =\mathbf{F}, \quad 0<\tau<\Delta \tau \\
\mathbf{w}(0) & =\mathbf{u}^{(0)}
\end{aligned}
$$

for $\Delta \tau>0$ called the smoothing step. The solution to (3) is

$$
\mathbf{w}(\Delta \tau)=e^{-L_{1} \Delta \tau} \mathbf{u}^{(0)}+\left(I_{1}-e^{-L_{1} \Delta \tau}\right) L_{1}^{-1} \mathbf{F},
$$

where $I_{1}$ indicates the identity matrix on $\Omega_{1}$. If the eigenvalues of $L_{1}$ have strictly positive real parts, $\|\mathbf{w}(\Delta \tau)-\mathbf{u}\|<\left\|\mathbf{u}^{(0)}-\mathbf{u}\right\|$ for any norm.

More generally from (4), we may define a smoothing technique for (2) as

$$
\begin{aligned}
& \mathbf{w}^{k}=S \mathbf{w}^{k-1}+\left(I_{1}-S\right) L_{1}^{-1} \mathbf{F}, \quad k=1, \ldots, \nu, \\
& \mathbf{w}^{0}=\mathbf{u}^{(0)}
\end{aligned}
$$


where the exponential smoother $S_{\exp }=e^{-L_{1} \Delta \tau}$ yields the dual time-marching procedure in (4). After $\nu$ steps, the iterative method (5) leads to

$$
\mathbf{w}=S^{\nu} \mathbf{u}^{(0)}+\left(I_{1}-S^{\nu}\right) L_{1}^{-1} \mathbf{F} .
$$

The procedure (6) converges if the spectral radius $\rho(S)$, i.e. the maximum among the absolute values of the eigenvalues, is less than one.

As a motivating example, consider the Jacobi method applied to the 2nd order discretization of the one-dimensional Poisson equation on $\Omega_{1}$ with meshsize $\Delta x$. The resulting smoother $S=I_{1}-\left(\Delta x^{2} / 4\right) L_{1}$ effectively damps high frequency errors while keeping smooth residuals substantially unchanged after a number of iterations $[2,14,15]$. This behavior justifies the introduction of grid coarsening to damp low frequency errors.

\subsection{Coarse-grid correction}

Next, consider the error $\mathbf{e}=L_{1}^{-1} \mathbf{F}-\mathbf{w}$ and the residual problem

$$
L_{1} \mathbf{e}=\mathbf{F}-L_{1} \mathbf{w}
$$

Rather than solving this system directly, we introduce a subset of $\Omega_{1}$, called the coarse grid $\Omega_{2}$, and solve the associated correction problem

$$
L_{2} \mathbf{d}=I_{r}\left(\mathbf{F}-L_{1} \mathbf{w}\right)
$$

on $\Omega_{2}$. The problem (8) is obtained from (7) by making use of

- a restriction operator $I_{r}: \Omega_{1} \rightarrow \Omega_{2}$,

- a coarse-grid operator $L_{2}: \Omega_{2} \rightarrow \Omega_{2}$.

The coarse-grid operator can be built by using the Galerkin condition [3]

$$
L_{2}=I_{r} L_{1} I_{p}
$$

where $I_{p}: \Omega_{2} \rightarrow \Omega_{1}$ is a prolongation operator. However, $L_{2}$ can also be built in other ways, for example by using the same discretization as $L_{1}$ on $\Omega_{2}$. In section 2.5, the main implications of using (9) are discussed in more detail.

A common choice for $I_{p}$ is based on linear interpolation. Assume for example that $\Omega_{1}=\left\{x_{j}: x_{j}=j \Delta x, j=0, \ldots, N\right\}$ with $\Delta x=1 / N$, and that $\Omega_{2}$ consists of the even nodes of $\Omega_{1}$. This leads to

$$
\left(I_{p} \mathbf{v}\right)_{m}=\left\{\begin{array}{ll}
v_{j}, & m=2 j, \\
\frac{1}{2}\left(v_{j}+v_{j+1}\right), & m=2 j+1,
\end{array} \quad j=0, \ldots, N / 2\right.
$$


while the restriction operator is usually given in terms of the prolongation operator as

$$
I_{r}=I_{p}^{T} / 2 .
$$

The choice (11), graphically represented in Figure 1, is justified by requiring that the two scalar products

$$
\left(\phi_{1}, \boldsymbol{\psi}_{1}\right)_{1}=\Delta x \boldsymbol{\phi}_{1}^{T} \boldsymbol{\psi}_{1}, \quad\left(\boldsymbol{\phi}_{2}, \boldsymbol{\psi}_{2}\right)_{2}=2 \Delta x \boldsymbol{\phi}_{2}^{T} \boldsymbol{\psi}_{2}
$$

on the fine- and coarse-grid respectively, are equal for $\boldsymbol{\phi}_{1}=I_{p} \boldsymbol{\phi}_{2}$ and $\boldsymbol{\psi}_{2}=$ $I_{r} \boldsymbol{\psi}_{1}$. As a consequence, the resulting interpolation operators $I_{r}$ and $I_{p}$ are adjoint to each other with respect to the scalar products $(\cdot, \cdot)_{1}$ and $(\cdot, \cdot)_{2}$, i.e. (11) follows.

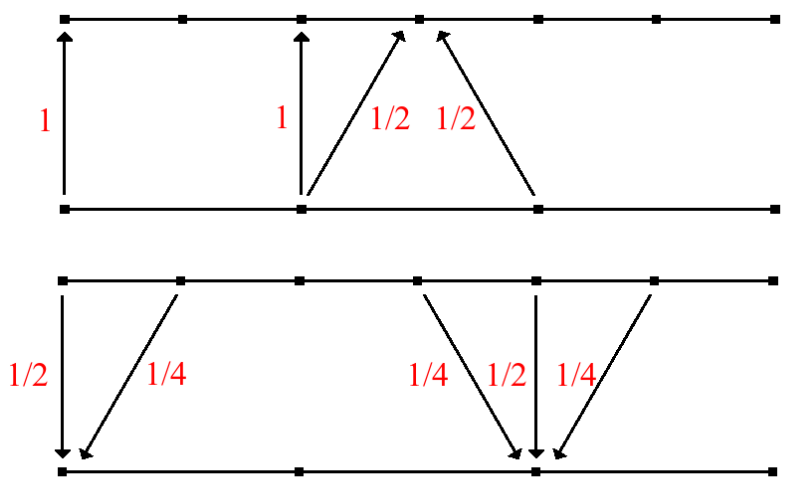

Figure 1: Graphical representation of the prolongation operator $I_{p}$ (top) in (10) and the restriction operator $I_{r}$ (bottom) in (11).

\subsection{Fine-grid update and the final multigrid iteration scheme}

Finally, we update the fine grid solution with the correction $\mathbf{d}$ as

$$
\mathbf{u}^{(1)}=\mathbf{w}+I_{p} \mathbf{d} .
$$

The relation (12), together with (6) and (8), provides an iterative method for solving (1):

$$
\mathbf{u}^{(n+1)}=M \mathbf{u}^{(n)}+N \mathbf{F},
$$

where

$$
M=C S^{\nu}, \quad C=I_{1}-I_{p} L_{2}^{-1} I_{r} L_{1} \quad \text { and } \quad N=\left(I_{1}-M\right) L_{1}^{-1} .
$$


We refer to $M$ as the multigrid iteration matrix and to $C$ as the coarse-grid correction operator.

The role of $M$ in the convergence of the iterative method (13) is central. By considering the errors $\mathbf{e}^{(n)}=\mathbf{u}^{(n)}-L_{1}^{-1} \mathbf{F}$, we get

$$
\mathbf{e}^{(n+1)}=M \mathbf{e}^{(n)},
$$

which leads to convergence if the spectral radius of $M, \rho(M)$, is less than 1 .

Remark 2.1. The spectral radius is the asymptotic convergence factor of the iteration. For $n$ sufficiently large we have $\left\|\mathbf{e}^{(n+1)}\right\| \leq \rho(M)\left\|\mathbf{e}^{(n)}\right\|[2]$.

\subsection{The coarse-grid correction operator}

In section 2.3 we claimed that the Galerkin condition (9) can be used to build a coarse-grid operator $L_{2}$. This choice leads to interesting theoretical properties of the coarse-grid correction operator $C$ in (14), as shown in [3]. To start with, we recall the following

Lemma 2.2. If the Galerkin condition (9) holds, then the coarse-grid correction operator $C$ in (14) is idempotent, i.e. $C^{k}=C$ for every $k \in \mathbb{N}$.

Proof. By multiplying $C$ by itself we get

$$
\begin{aligned}
C^{2} & =\left(I_{1}-I_{p} L_{2}^{-1} I_{r} L_{1}\right)\left(I_{1}-I_{p} L_{2}^{-1} I_{r} L_{1}\right) \\
& =I_{1}-2 I_{p} L_{2}^{-1} I_{r} L_{1}+I_{p} L_{2}^{-1} I_{r} L_{1} I_{p} L_{2}^{-1} I_{r} L_{1}=C
\end{aligned}
$$

since the Galerkin condition $L_{2}=I_{r} L_{1} I_{p}$ is fulfilled.

The idempotency of $C$ implies that it is a projection matrix with eigenvalues equal to zeros and ones only [16]. Moreover,

Corollary 2.3. If the Galerkin condition (9) holds, the image of the prolongation operator $I_{p}, \operatorname{Im}\left(I_{p}\right)$, is contained in the nullspace of $C$.

Proof. Consider a vector $\mathbf{y} \in \operatorname{Im}\left(I_{p}\right)$, i.e. $\mathbf{y}=I_{p} \mathbf{z}$. When the coarse-grid correction operator acts on this vector, we find

$$
C \mathbf{y}=\left(I_{1}-I_{p} L_{2}^{-1} I_{r} L_{1}\right) I_{p} \mathbf{z}=I_{p} \mathbf{z}-I_{p} L_{2}^{-1} I_{r} L_{1} I_{p} \mathbf{z}=\mathbf{0},
$$

since the Galerkin condition holds.

Remark 2.4. Corollary 2.3 implies that $C$ cancels all grid functions which can be represented through the prolongation operator $I_{p}$. This indicates that $C$ deals with smooth parts of the error while $S$ damps high frequency modes. 


\section{The SBP-SAT discretization}

In this section we introduce the SBP-SAT technique [10]. For simplicity, we only consider equidistant grids $\mathbf{x}=\left[x_{0}, \ldots, x_{N}\right]^{T}$ which are conforming to the domain boundaries, although other options exist, see [17, 18, 19].

Definition 3.1. $D_{1}=P^{-1} Q$ is a $(p, q)$-accurate first derivative SBP operator if i) it differentiates $x^{k}$ exactly for $k=0, \ldots, p$ in the interior and $k=$ $0, \ldots, q$ at the boundaries, ii) $P$ is a symmetric positive definite matrix, iii) $Q+Q^{T}=B=\operatorname{diag}(-1,0, \ldots, 0,1)$.

Condition ii) defines a scalar product and a norm by means of

$$
(\phi, \psi)_{P}=\phi^{T} P \boldsymbol{\psi}, \quad\|\phi\|_{P}=\sqrt{(\phi, \phi)_{P}},
$$

while condition iii) causes $D_{1}$ to mimic the integration-by-parts rule since

$$
\left(\boldsymbol{\phi}, D_{1} \boldsymbol{\psi}\right)_{P}=\phi_{N} \psi_{N}-\phi_{0} \psi_{0}-\left(D_{1} \boldsymbol{\phi}, \boldsymbol{\psi}\right)_{P}
$$

SBP operators based on centered finite-differences and diagonal norms $P$ are available for even orders $p=2 q$ in the interior, while the boundary closure is $q$ th order accurate. We will for stability reasons [20, 21, 22] only consider operators with a diagonal $P$.

Narrow-diagonal second-derivative SBP operators [23, 24] for diagonal norms $P$ can be also defined.

Definition 3.2. $D_{2}=P^{-1}\left(-S^{T} A+B\right) S$ is said to be a $(2 q, q)$ th-order accurate compact second-derivative $S B P$ operator if, for any smooth grid function $\mathbf{u}$, i) $D_{2} \mathbf{u}=u_{x x}+\mathcal{O}\left(h^{2 q}\right)$ in the interior, ii) $D_{2} \mathbf{u}=u_{x x}+\mathcal{O}\left(h^{q}\right)$ at the boundaries, iii) $[S \mathbf{u}]_{i}=u_{x}\left(x_{i}\right)+\mathcal{O}\left(h^{q+1}\right)$ for $i \in\{0, N\}$, iv) $A+A^{T} \geq 0$.

Remark 3.3. The accuracy requirements on $D_{2}$ can be specified in the same form as for $D_{1}$ (cf. Definition 3.1). In particular, a 2qth-order accurate SBP operator for the second derivative is such that: i) $D_{2}$ exactly mimics the second derivative of $x^{k}$ for $k=0, \ldots, 2 q+1$ in the interior and for $k=0, \ldots, q+1$ at the boundaries; ii) $S$ differentiates $x^{k}$ exactly for $k=$ $0, \ldots, q+1$ at the boundary nodes $x_{0}$ and $x_{N}$.

Remark 3.4. It is also possible to build second-derivative SBP operators by applying the first derivative operator twice as $D_{2}=D_{1} D_{1}$, which gives $S=D_{1}$ and $A=D_{1}^{T} P D_{1}$. However, this choice does not modify modes with the Nyquist frequency (the highest frequency that can exist on the grid) [24]. 
The second derivative operator $D_{2}$ satisfies the following summation-byparts property

$$
\left(\boldsymbol{\phi}, D_{2} \boldsymbol{\psi}\right)_{P}=\phi_{N}(S \boldsymbol{\psi})_{N}-\phi_{0}(S \boldsymbol{\psi})_{0}-(S \boldsymbol{\phi})^{T} A(S \boldsymbol{\psi})
$$

SBP operators together with a strong treatment of the boundary conditions only admits stability proofs for very simple problems. This was shown in [25], where the weak SAT technique was proposed to complement the SBP schemes. By discretizing an initial boundary value problem augmented with well-posed boundary conditions using the SBP-SAT approach leads to a stable semi-discrete problem. For comprehensive reviews on the SBP-SAT technique, see $[10,26]$.

\subsection{An example of the SBP-SAT technique}

Consider the advection problem

$$
\begin{aligned}
u_{t}+u_{x} & =0, & 0<x<1, \quad t>0, \\
u(0, t) & =g(t), & t>0 \\
u(x, 0) & =h(x), & 0<x<1,
\end{aligned}
$$

where both $g$ and $h$ are known data. The problem (18) has an energyestimate and is well-posed, since the analytical solution is a right traveling wave, and the boundary condition is imposed at the left boundary.

Consider an $(N+1)$-point uniform grid on [0,1], given by $x_{j}=j \Delta x$ for $j=0, \ldots, N$, where $\Delta x=1 / N$. We introduce the grid function $h_{j}=h\left(x_{j}\right)$ and to each grid point associate the approximate solution $u_{j}$. By applying the SBP-SAT discretization in space to (18), we get

$$
\begin{aligned}
\mathbf{u}_{t}+D_{1} \mathbf{u} & =P^{-1} \sigma\left(u_{0}-g\right) \mathbf{e}_{0}, \quad t>0 \\
\mathbf{u}(0) & =\mathbf{h}
\end{aligned}
$$

where $\mathbf{u}=\left[u_{0}, \ldots, u_{N}\right]^{T}, \mathbf{h}=\left[h_{0}, \ldots, h_{N}\right]^{T}, \sigma \in \mathbb{R}$ is a penalty parameter which can be tuned for stability, and $\mathbf{e}_{0}=[1,0, \ldots, 0]^{T} \in \mathbb{R}^{N+1}$. We want to find $\sigma$ such that the problem (19) is strongly stable, i.e. such that

$$
\|\mathbf{u}(t)\|^{2} \leq K(t)\left(\|\mathbf{h}\|^{2}+\max _{\tau \in[0, t]}|g(\tau)|^{2}\right)
$$

in a suitable norm. In (20), $K(t)$ is independent of the data and bounded for any finite $t$ and meshsize $\Delta x$ (for further details, see $[10,12]$ ). 
By applying the energy method (multiplying equation (19) with $\mathbf{u}^{T} P$ and adding the transpose) in combination with the SBP property (16) we find

$$
\frac{\mathrm{d}}{\mathrm{d} t}\|\mathbf{u}\|_{P}^{2}=-\frac{\sigma^{2}}{1+2 \sigma} g^{2}-u_{N}^{2}+\frac{\left[(1+2 \sigma) u_{0}-\sigma g\right]^{2}}{1+2 \sigma},
$$

which, by time-integration, lead to an estimate of the form (20) for $\sigma<-1 / 2$.

\section{A new coarse-grid correction for summation-by-parts schemes}

The use of the Galerkin condition (9) together with the prolongation and restriction operators in (10) and (11) leads to inaccurate coarse-grid operators that do not mimic the integration-by-parts rule. It is well known that the accuracy of the coarse-grid discretizations does not affect the accuracy of the steady-state solution, if the multigrid method converges. On the other hand, an accurate representation of the coarse-grid correction problem (8) should reduce the errors due to grid coarsening and could possibly improve the multigrid convergence rate.

In order to accurately represent the coarse-grid correction problem, a more suitable class of interpolation operators is required. To avoid ambiguity, henceforth the operators related to the fine- and coarse-grid will be denoted with the index 1 and 2, respectively.

\subsection{Summation-by-parts preserving interpolation}

Consider the following steady model problem

$$
\begin{aligned}
u_{x} & =f, \quad 0<x<1, \\
u(0) & =g .
\end{aligned}
$$

By applying the SBP-SAT method on an equidistant grid $\Omega_{1}$ with meshsize $\Delta x$ we get $L_{1} \mathbf{u}=\mathbf{F}$, where $L_{1}=D_{1,1}-P_{1}^{-1} \sigma \mathbf{e}_{0} \mathbf{e}_{0}^{T}$ and $\mathbf{F}=\mathbf{f}-\sigma P_{1}^{-1} \mathbf{e}_{0} g$. Since our problem is written on the form (2), we can apply the multigrid algorithm in section 2 directly. Note that the time-marching smoothing procedure (3) yields the semi-discrete problem (19), which for $\sigma<-1 / 2$ is stable.

As in section 2.3, the coarse-grid consists of the even nodes of the finegrid, i.e. $\Omega_{2}=\left\{x_{j}: x_{j}=2 j \Delta x, j=0, \ldots, N / 2\right\}$. However, the restriction operator chosen in (11) does not produce a coarse-grid operator $L_{2}$ consistent with the SBP property (16). To overcome this issue, we rather consider

$$
I_{r}=P_{2}^{-1} I_{p}^{T} P_{1}
$$


which was first introduced in [9]. The relation (22) is obtained by enforcing

$$
\left(I_{p} \boldsymbol{\xi}_{2}, \boldsymbol{\xi}_{1}\right)_{P_{1}}=\left(\boldsymbol{\xi}_{2}, I_{r} \boldsymbol{\xi}_{1}\right)_{P_{2}}
$$

where the SBP-based scalar products are defined in (15).

By using (22), it is possible to build pairs of consistent and accurate prolongation and restriction operators. The following definition of the so called SBP preserving interpolation operators in (22) was given in [9], where the operators were used to couple SBP-SAT formulations on grids with different meshsizes in a stable manner.

Definition 4.1. Let the row-vectors $\mathbf{x}_{1}^{k}$ and $\mathbf{x}_{2}^{k}$ be the projections of the monomials $x^{k}$ onto equidistant $1-D$ grids corresponding to a fine and coarse grid, respectively. We say that $I_{r}$ and $I_{p}$ in (22) are 2qth-order accurate $S B P$-preserving interpolation operators if $I_{r} \mathbf{x}_{1}^{k}-\mathbf{x}_{2}^{k}$ and $I_{p} \mathbf{x}_{2}^{k}-\mathbf{x}_{1}^{k}$ vanish for $k=0, \ldots, 2 q-1$ in the interior and for $k=0, \ldots, q-1$ at the boundaries.

In [27] it was shown that the sum of the orders of the prolongation and of the restriction operators should at least be equal to the order of the differential equation. As a consequence, the use of high-order interpolation is not required in order to solve (21) with multigrid. However, high-order grid transfer operators can without drawbacks be used in combination with high-order discretizations, see for example [28].

SBP-preserving interpolation operators with minimal bandwidth are given in Appendix A for 2nd, 4th and 6th order accurate operators in the interior. Note that the 2nd order accurate operator $I_{p}$ coincides with the prolongation operator (10) and correctly represents first order polynomials for every fine-grid node. The corresponding restriction operator $I_{r}$, which differs from the conventional one in (11) at the boundary nodes, is shown in Figure 2. It is, in contrast to the one in Figure 1, consistent at the boundaries.

\subsection{SBP-preserving interpolation applied to the first derivative}

Next, we study the correction problem (8) with the coarse-grid operator $L_{2}$ given by the Galerkin condition (9) and the SBP-preserving operators in Definition 4.1. Initially, we only consider the first derivative fine-grid SBP operator $D_{1,1}$ and its coarse-grid counterpart $D_{1,2}=I_{r} D_{1,1} I_{p}$.

Proposition 4.2. The interpolation operators in (22) lead to a coarse-grid first derivative $D_{1,2}$ with the summation-by-parts property (16). 


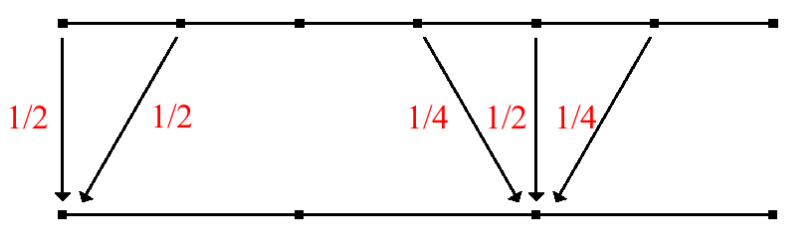

Figure 2: The 2nd-order accurate SBP-preserving restriction operator $I_{r}$ (cf. Figure 1).

Proof. To start with, we rewrite the left hand-side of (16) for $D_{1,2}$ and two coarse-grid functions $\boldsymbol{\phi}_{2}$ and $\boldsymbol{\psi}_{2}$ by using the adjoint relation (23)

$$
\left(\boldsymbol{\phi}_{2}, D_{1,2} \boldsymbol{\psi}_{2}\right)_{P_{2}}=\left(\boldsymbol{\phi}_{2}, I_{r}\left(D_{1,1} I_{p} \boldsymbol{\psi}_{2}\right)\right)_{P_{2}}=\left(I_{p} \boldsymbol{\phi}_{2}, D_{1,1}\left(I_{p} \boldsymbol{\psi}_{2}\right)\right)_{P_{1}} \text {. }
$$

Next, the SBP property for the fine-grid operator $D_{1}$ leads to

$$
\left(\boldsymbol{\phi}_{2}, D_{1,2} \boldsymbol{\psi}_{2}\right)_{P_{2}}=\left(I_{p} \boldsymbol{\phi}_{2}\right)_{N}\left(I_{p} \boldsymbol{\psi}_{2}\right)_{N}-\left(I_{p} \boldsymbol{\phi}_{2}\right)_{0}\left(I_{p} \boldsymbol{\psi}_{2}\right)_{0}-\left(D_{1,1}\left(I_{p} \boldsymbol{\phi}_{2}\right), I_{p} \boldsymbol{\psi}_{2}\right)_{P_{1}} \text {. }
$$

Since both grids are conforming to the domain boundaries, the prolongation onto the boundary nodes of the fine-grid is exact (cf. Appendix A). Furthermore, by applying (23) to the right hand-side of (24), we find

$$
\left(\boldsymbol{\phi}_{2}, D_{1,2} \boldsymbol{\psi}_{2}\right)_{P_{2}}=\phi_{2, N / 2} \psi_{2, N / 2}-\phi_{2,0} \psi_{2,0}-\left(D_{1,2} \boldsymbol{\phi}_{2}, \boldsymbol{\psi}_{2}\right)_{P_{2}}
$$

and the claim follows.

The coarse-grid first derivative $\mathrm{SBP}$ operator $D_{1,2}$ retains the order of accuracy of the original scheme at the interior nodes, if $2 q$ th order SBPpreserving interpolators are used. In particular, we can prove

Proposition 4.3. Consider the $2 q$ th-order accurate SBP-preserving interpolation operators $I_{p}$ and $I_{r}$. If $D_{1,1}$ is a $(2 q, q)$-first derivative SBP operator on the fine-grid, then $D_{1,2}$ is 2qth-order accurate in the interior nodes and at least $(q-1)$ th-order accurate close to the boundaries.

Proof. See Appendix B.

Remark 4.4. The accuracy result for the interior nodes was conjectured in [9], but not proved. 
Remark 4.5. For $q=1$ the coarse grid operator $D_{1,2}$ has the same structure as $D_{1,1}$ and is first-order accurate also at the boundaries. If $q>1$ the operator $D_{1,2}$ has a wider stencil and boundary closure than $D_{1,1}$.

Consider now the fine-grid operator $L_{1}=D_{1,1}-\sigma P_{1}^{-1} \mathbf{e}_{0} \mathbf{e}_{0}^{T}$ and the forcing function $\mathbf{F}=\mathbf{f}-\sigma P_{1}^{-1} \mathbf{e}_{0} g$ associated to the SBP-SAT discretization of (21). The corresponding residual problem (7) becomes

$$
D_{1,1} \mathbf{e}=\mathbf{f}-D_{1,1} \mathbf{w}+\sigma P_{1}^{-1} \mathbf{e}_{0}\left[\mathbf{e}_{0}^{T} \mathbf{e}-\left(g-w_{0}\right)\right],
$$

where $w_{0}=\mathbf{e}_{0}^{T} \mathbf{w}$. The Galerkin condition (9) in combination with the interpolation operators in (22) leads to

$$
L_{2}=I_{r} L_{1} I_{p}=I_{r} D_{1,1} I_{p}-\sigma I_{r} P_{1}^{-1} \mathbf{e}_{0} \mathbf{e}_{0}^{T} I_{p}=D_{1,2}-\sigma P_{2}^{-1}\left(I_{p}^{T} \mathbf{e}_{0}\right)\left(I_{p}^{T} \mathbf{e}_{0}\right)^{T},
$$

and the coarse-grid correction problem (8) becomes

$$
D_{1,2} \mathbf{d}=I_{r}\left(\mathbf{f}-D_{1,1} \mathbf{w}\right)+\sigma P_{2}^{-1}\left(I_{p}^{T} \mathbf{e}_{0}\right)\left[\left(I_{p}^{T} \mathbf{e}_{0}\right)^{T} \mathbf{d}-\left(g-w_{0}\right)\right] .
$$

Since $I_{p}^{T} \mathbf{e}_{0}$ is the counterpart of $\mathbf{e}_{0}$ on the coarse-grid, i.e. it projects any coarse-grid function onto the first node, (25) and (26) have the same SBPSAT structure on different grids. In particular, the coarse-grid correction problem (8) is consistent and high-order accurate in the interior nodes.

Remark 4.6. If the time-dependent version of the fine-grid problem (25) is stable, so is the coarse-grid problem (26). In particular, the penalty parameter remains the same.

\subsection{SBP-preserving interpolation applied to the second derivative}

The SBP-preserving interpolation can also be applied to the second derivative operator. We end the section by showing that the coarse-grid operator, $D_{2,2}=I_{r} D_{2,1} I_{p}$, is an SBP operator for the second derivative which retains the accuracy of the fine-grid operator $D_{2,1}$ at the interior nodes. First, we prove

Proposition 4.7. The interpolation operators in (22) lead to a coarse-grid second derivative operator $D_{2,2}$ which preserves the summation-by-parts property (17). 
Proof. As in the proof of Proposition 4.2, we rewrite the left hand-side of (17) for $D_{2,2}$ and two coarse-grid functions $\boldsymbol{\phi}_{2}$ and $\boldsymbol{\psi}_{2}$ by using (23)

$$
\left(\boldsymbol{\phi}_{2}, D_{2,2} \boldsymbol{\psi}_{2}\right)_{P_{2}}=\left(\boldsymbol{\phi}_{2}, I_{r} D_{2,1} I_{p} \boldsymbol{\psi}_{2}\right)_{P_{2}}=\left(I_{p} \boldsymbol{\phi}_{2}, D_{2,1} I_{p} \boldsymbol{\psi}_{2}\right)_{P_{1}} .
$$

By applying the SBP property (17) for the fine-grid second derivative $D_{2,1}$, we find

$\left(\boldsymbol{\phi}_{2}, D_{2,2} \boldsymbol{\psi}_{2}\right)_{P_{2}}=\left(I_{p} \boldsymbol{\phi}_{2}\right)_{N}\left(S I_{p} \boldsymbol{\psi}_{2}\right)_{N}-\left(I_{p} \boldsymbol{\phi}_{2}\right)_{0}\left(S I_{p} \boldsymbol{\psi}_{2}\right)_{0}-\left(S I_{p} \boldsymbol{\phi}_{2}\right)^{T} A\left(S I_{p} \boldsymbol{\psi}_{2}\right)$.

Both the fine- and coarse-grid are conforming to the domain boundaries, implying that $\left(I_{p} \boldsymbol{\phi}_{2}\right)_{i}=\phi_{2, i / 2}$ and $\left(S I_{p} \boldsymbol{\phi}_{2}\right)_{i}=\left(S \boldsymbol{\phi}_{2}\right)_{i / 2}$ for $i \in\{0, N\}$. Thus

$$
\left(\boldsymbol{\phi}_{2}, D_{2,2} \boldsymbol{\psi}_{2}\right)_{P_{2}}=\phi_{2, N / 2}\left(S \boldsymbol{\phi}_{2}\right)_{N / 2}-\phi_{2,0}\left(S \boldsymbol{\phi}_{2}\right)_{0}-\left(S I_{p} \boldsymbol{\phi}_{2}\right)^{T} A\left(S I_{p} \boldsymbol{\psi}_{2}\right)
$$

mimics the continuous integration-by-parts rule for the second derivative.

Moreover, an accuracy result similar to Proposition 4.3 for $D_{2,2}$ is

Proposition 4.8. Consider the 2qth-order accurate SBP-preserving interpolation operators $I_{p}$ and $I_{r}$. If $D_{2,1}$ is a $(2 q, q)$ th-order accurate second derivative $S B P$ operator on the fine-grid, then $D_{2,2}$ is a $(2 q, q-2)$ th order second derivative SBP operator on the coarse grid.

Proof. See Appendix B.

Remark 4.9. For $q=1$ the coarse grid operator $D_{2,2}$ has zeros on the first and last row, while its stencil is the same as $D_{2,1}$ except for the scaling factor, proportional to the gridsize. Thus, $D_{2,2}$ is a $(2,0)$ th order accurate representation of the second derivative (cf. Remark 3.3). Similarly to the first derivative, if $q>1$ the operator $D_{2,2}$ has a wider stencil and boundary closure than $D_{2,1}$.

Proposition 4.8 implies that also differential problems involving second derivatives can be accurately represented on each grid level by using the Galerkin condition (9) and the SBP-preserving interpolation (22).

\section{Numerical experiments}

In this section we consider multigrid iteration schemes for relevant model problems on a square domain. To simplify the notation, we refer to the order of the discretizations by the order of the SBP operators on the interior nodes. Hence, the performances of the conventional interpolation (10),(11) for $2 q$ thorder accurate discretizations are compared with the ones of the $2 q$ th-order SBP-preserving grid transfer operators. 


\subsection{An elliptic equation}

Since multigrid methods have proven to be very efficient for elliptic boundary value problems [2], we start by considering

$$
\begin{aligned}
& -\alpha u_{x x}-\beta u_{y y}=f, \quad 0<x<1, \quad 0<y<1 \text {, } \\
& \left(u-u_{x}\right)(0, y)=g_{W}(y), \quad 0<y<1 \text {, } \\
& \left(u+u_{y}\right)(x, 1)=g_{N}(x), \quad 0<x<1, \\
& \left(u+u_{x}\right)(1, y)=g_{E}(y), \quad 0<y<1 \text {, } \\
& \left(u-u_{y}\right)(x, 0)=g_{S}(x), \quad 0<x<1
\end{aligned}
$$

with $\alpha, \beta$ positive constants and $f, g_{W}, g_{N}, g_{E}, g_{S}$ known data. The boundary conditions in (27) are chosen in order to make the augmented problem in dual time $u_{\tau}-\alpha u_{x x}-\beta u_{y y}=f$ strongly well-posed. Note that (27) represents the Poisson equation for $\alpha=\beta=1$.

To discretize (27), we consider a grid with $N_{x}+1$ and $N_{y}+1$ equidistant nodes $x_{j}, y_{k}$ in the $x$ - and $y$-direction, respectively. Also, let

$$
\mathbf{f}=\left[f\left(x_{0}, y_{0}\right), f\left(x_{0}, y_{1}\right), \ldots, f\left(x_{0}, y_{N_{y}}\right), f\left(x_{1}, y_{0}\right), \ldots, f\left(x_{N_{x}}, y_{N_{y}}\right)\right]^{T},
$$

while $\mathbf{u}$ is the approximate solution, with the same arrangement. The vector $\mathbf{u}$ is the solution to the following SBP-SAT formulation

$$
\begin{aligned}
-\alpha\left(D_{2 x} \otimes I_{y}\right) \mathbf{u}-\beta\left(I_{x} \otimes D_{2 y}\right) \mathbf{u}=\mathbf{f} & -\alpha\left(P_{x}^{-1} E_{0 x} \otimes I_{y}\right)\left[\left(\left(I_{x}-S_{x}\right) \otimes I_{y}\right) \mathbf{u}-\mathbf{g}_{W}\right] \\
& -\beta\left(I_{x} \otimes P_{y}^{-1} E_{N y}\right)\left[\left(I_{x} \otimes\left(I_{y}+S_{y}\right)\right) \mathbf{u}-\mathbf{g}_{N}\right] \\
& -\alpha\left(P_{x}^{-1} E_{N x} \otimes I_{y}\right)\left[\left(\left(I_{x}+S_{x}\right) \otimes I_{y}\right) \mathbf{u}-\mathbf{g}_{E}\right] \\
& -\beta\left(I_{x} \otimes P_{y}^{-1} E_{0 y}\right)\left[\left(I_{x} \otimes\left(I_{y}-S_{y}\right)\right) \mathbf{u}-\mathbf{g}_{S}\right] .
\end{aligned}
$$

The data $g_{W}, g_{N}, g_{E}$ and $g_{S}$ are injected at the appropriate grid points. In (28), the symbol $\otimes$ denotes the Kronecker product defined by

$$
A \otimes C=\left[\begin{array}{ccc}
a_{11} C & \cdots & a_{1 n} C \\
\vdots & \ddots & \vdots \\
a_{m 1} C & \cdots & a_{m n} C
\end{array}\right] \in \mathbb{R}^{m r \times n s}, \quad A=\left\{a_{i j}\right\} \in \mathbb{R}^{m \times n}, \quad C \in \mathbb{R}^{r \times s}
$$

while the subscripts $x, y$ refer to the coordinate directions. Moreover, $E_{0 i}=$ $\operatorname{diag}(1,0, \ldots, 0)$ and $E_{N i}=\operatorname{diag}(0, \ldots, 0,1), i \in\{x, y\}$, are $\left(N_{i}+1\right)$-square matrices. 
The problem (28) can be written in compact form as $L_{1} \mathbf{u}=\mathbf{F}$ and solved by using the two-level multigrid method described in section 2, with both $N_{x}$ and $N_{y}$ being even. Since the auxiliary problem

$$
\begin{aligned}
\mathbf{w}_{\tau}+L_{1} \mathbf{w} & =\mathbf{F}, \quad \tau>0 \\
\mathbf{w}(0) & =\mathbf{w}^{(0)}
\end{aligned}
$$

is strongly stable, a smoothing procedure based on time-marching is conditionally stable. Well-posedness for (27) and stability in dual time for (29) are shown in Appendix C.

Finally, the grid transfer operators needed for multigrid are implemented by means of the Kronecker product defined above. Let $I_{r x}, I_{r y}, I_{p x}, I_{p y}$ be

the restriction and prolongation operators in the $x$ - and $y$-direction. The restriction and prolongation operators on the two-dimensional grid are given by $I_{r}=I_{r x} \otimes I_{r y}$ and $I_{p}=I_{p x} \otimes I_{p y}$, respectively.

\subsubsection{The Poisson equation}

Consider $\alpha=\beta=1$ and a uniform grid such that $N_{x}=N_{y}=60$. The multigrid method is applied to (28) by using a third-order Runge-Kutta (RK3) time integrator as smoother, with $\nu=5$ smoothing steps per grid cycle. The multigrid iteration is studied as a function of the CFL number, which is equal to $\Delta \tau /(\Delta x)^{2}$. The forcing function and boundary data are given by the manufactured solution $v(x, y)=\cos (\pi(3 x-y)) e^{-x-y}$.

We consider a $2 \mathrm{nd}, 4$ th and 6th order accurate discretization of (28). In Figure 3, 4, 5 the logarithm of the spectral radius of the multigrid iteration matrix $M$ is shown as a function of the CFL number (left figure), together with the $\left(P_{x} \otimes P_{y}\right)$-norm of the error (right figure) for: i) the single-grid time integration (green triangles), ii) the two-grids iteration with SBP-preserving interpolation (blue circles), iii) the two-grids iteration with conventional interpolation (red crosses). The convergence is shown with the optimal CFL for each procedure, i.e. the CFL that minimizes the spectral radius of the correspondent iteration matrix. The SBP-preserving interpolation clearly improves the performances of the multigrid method for all cases.

To investigate the influence of different smoothers, we consider two additional smoothing techniques. Table 1 provides the asymptotic convergence factors $\rho(M)$ for multigrid procedures with RK3, Weighted Jacobi (WJ) and Successive Over Relaxation method (SOR). The values of $\rho(M)$ for each smoothing techniques are computed with the optimal choices of parameters, 

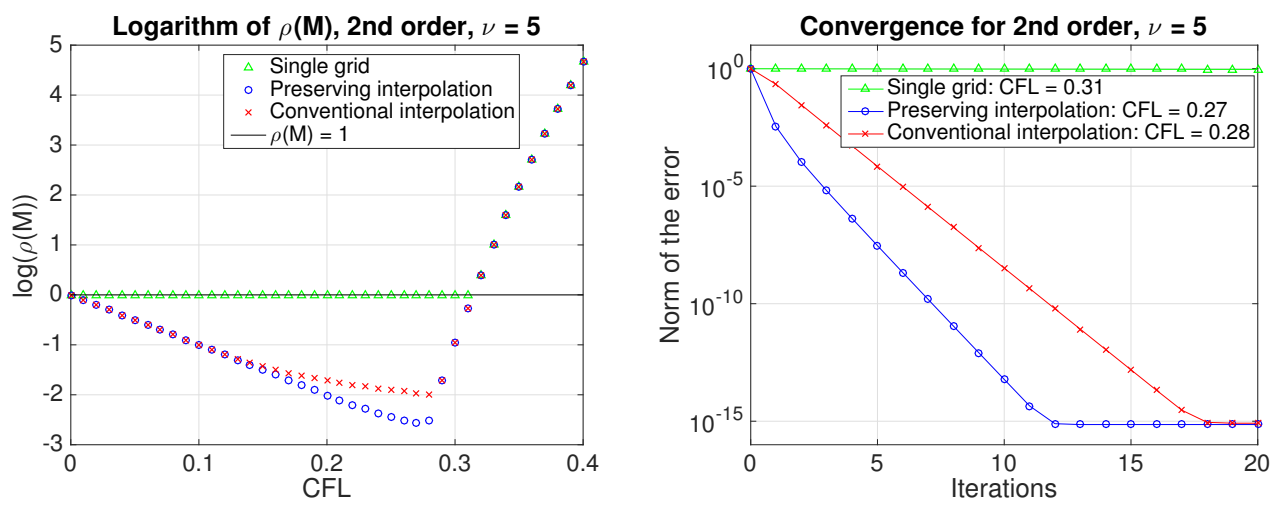

Figure 3: The left picture shows the logarithm of the spectral radius for a 2nd-order accurate discretization with $\nu=5$ steps of RK3. To the right, the history of convergence of the multigrid method with CFL $=0.31$ (single-grid), 0.27 (SBP-preserving interpolation), 0.28 (conventional interpolation).
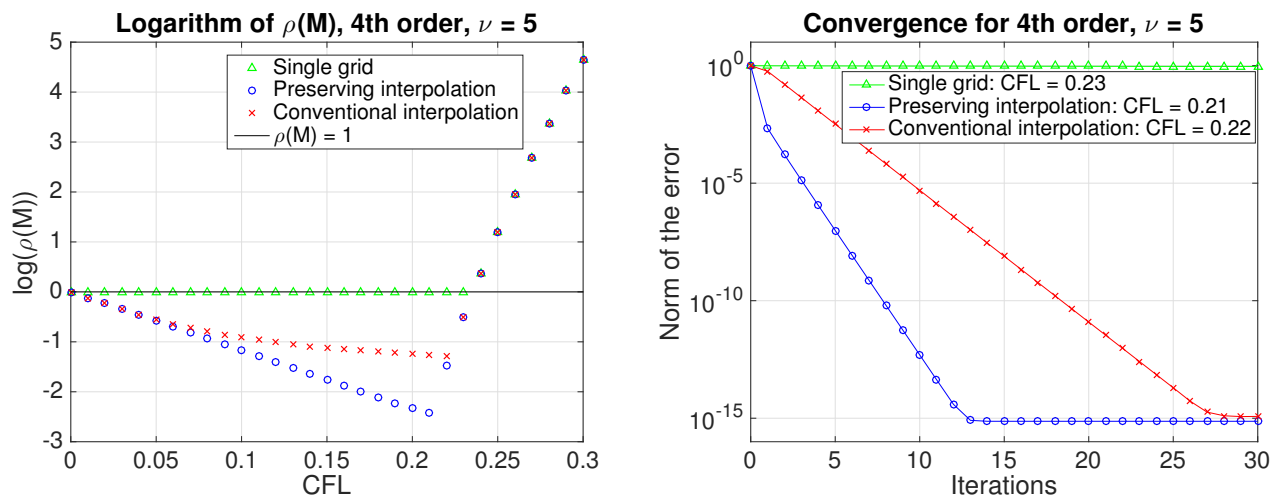

Figure 4: The left picture shows the logarithm of the spectral radius for a 4th-order accurate discretization with $\nu=5$ steps of RK3. To the right, the history of convergence of the multigrid method with $\mathrm{CFL}=0.23$ (single-grid), 0.21 (SBP-preserving interpolation), 0.22 (conventional interpolation). 

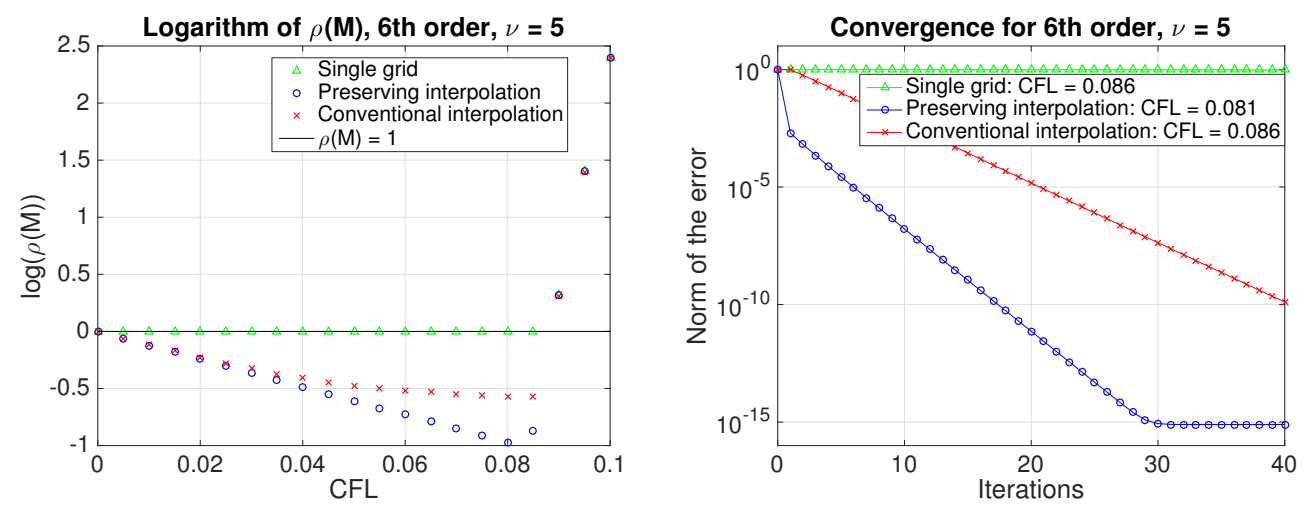

Figure 5: The left picture shows the logarithm of the spectral radius for a 6th-order accurate discretization with $\nu=5$ steps of RK3. To the right, the history of convergence of the multigrid method with CFL $=0.086$ (single-grid), 0.081 (SBP-preserving interpolation), 0.086 (conventional interpolation).

\begin{tabular}{|c|cc|cc|cc|}
\hline \hline$(\alpha, \beta)=(1,1)$ & C,2nd & P,2nd & C,4th & P,4th & C,6th & P,6th \\
\hline RK3 & 0.1373 & 0.0763 & 0.2775 & 0.0823 & 0.5622 & 0.3744 \\
WJ & 0.1500 & 0.0896 & 0.2631 & 0.1152 & 0.4908 & 0.3395 \\
SOR & 0.0724 & 0.0236 & 0.1513 & 0.0176 & 0.4003 & 0.0188 \\
\hline \hline
\end{tabular}

Table 1: Asymptotic convergence factor $\rho(M)$ with $\nu=5$ and optimal choice of coefficients for 3rd-order Runge-Kutta (RK3), Weighted Jacobi (WJ) and Successive Over Relaxation method (SOR) [29]. The conventional interpolation (C) is compared with the preserving grid transfer operators $(\mathrm{P})$.

numerically estimated as above. In all cases, the SBP-preserving interpolation leads to superior convergence. Furthermore, the SOR multigrid method has excellent perfomances in combination with the SBP-preserving interpolation. The behavior of the multigrid procedure with the SOR is shown in Figure 6 for the 6th-order discretization and different choices of the relaxation parameter $\omega$.

\subsubsection{The anisotropic elliptic problem}

Next, the multigrid procedure is studied for anisotropic problems, i.e. for $\alpha \neq \beta$. The standard multigrid method for this problem often leads to unsatisfactory results (which can sometimes be improved by means of a frequency decomposition approach [30]). Here, we want to compare the two 

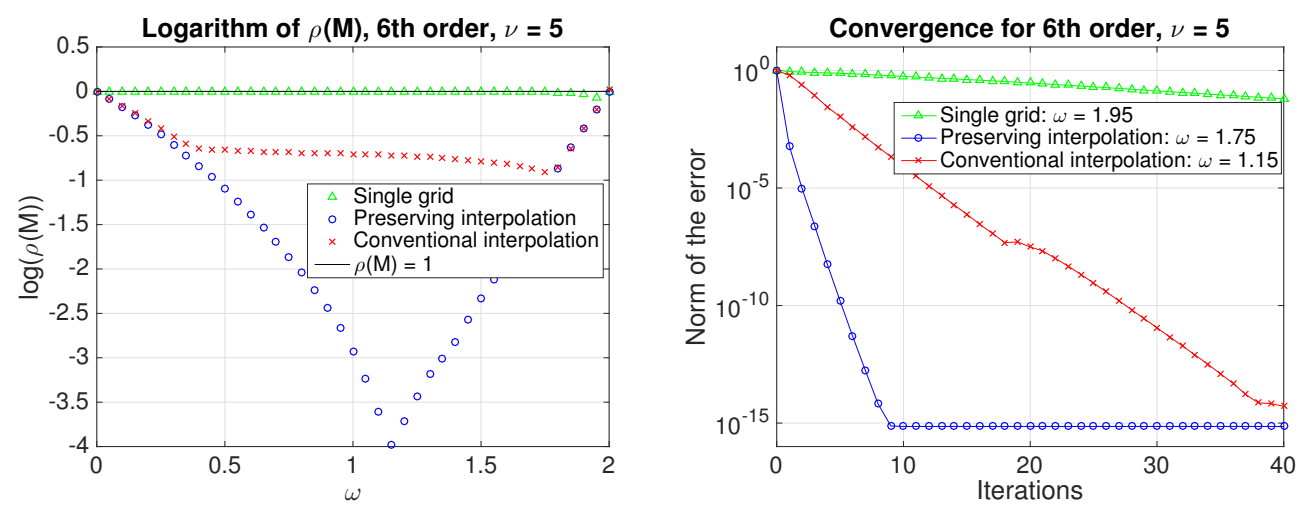

Figure 6: The left picture shows the logarithm of the spectral radius for a 6th-order accurate discretization with $\nu=5$ steps of SOR. To the right, the history of convergence of the multigrid method with $\omega=1.95$ (single-grid), 1.15 (SBP-preserving interpolation), 1.75 (conventional interpolation).

classes of interpolation operators for the standard multigrid algorithm.

In Table 2, we computed the spectral radius of the multigrid iteration matrix for RK3, WJ and SOR with optimal parameter choices, different orders of discretization, $\alpha=1$ and $\beta \in\{1 / 2,1 / 4,1 / 8\}$. As for the isotropic case, the SOR-based multigrid method has excellent perfomances in combination with the SBP-preserving interpolation. Moreover, the improvements on the convergence rates are valid for all the smoothers considered.

\subsection{The advection-diffusion problem}

Next, we add a hyperbolic character to the Poisson equation and consider the advection-diffusion problem

$$
\begin{aligned}
a u_{x}+b u_{y} & =\varepsilon\left(u_{x x}+u_{y y}\right)+f, & & 0<x<1, \quad 0<y<1, \\
\left(a u-\varepsilon u_{x}\right)(0, y) & =g_{W}(y), & & 0<y<1, \\
u_{y}(x, 1) & =g_{N}(x), & & 0<x<1, \\
u_{x}(1, y) & =g_{E}(y), & & 0<y<1, \\
\left(b u-\varepsilon u_{y}\right)(x, 0) & =g_{S}(x), & & 0<x<1 .
\end{aligned}
$$

In (30), $a, b, \varepsilon$ are positive constants and $f, g_{W}, g_{N}, g_{E}, g_{S}$ known data. Again, the boundary conditions in (30) are chosen in order to make the augmented problem in dual time strongly well-posed. The SBP-SAT dis- 


\begin{tabular}{|c|cc|cc|cc|}
\hline \hline$(\alpha, \beta)=(1,1 / 2)$ & C,2nd & P,2nd & C,4th & P,4th & C,6th & P,6th \\
\hline RK3 & 0.1865 & 0.1485 & 0.3271 & 0.1826 & 0.6002 & 0.5087 \\
WJ & 0.2096 & 0.1887 & 0.3285 & 0.2370 & 0.5281 & 0.4616 \\
SOR & 0.0773 & 0.0314 & 0.1527 & 0.0284 & 0.4091 & 0.0297 \\
\hline \hline \hline$(\alpha, \beta)=(1,1 / 4)$ & C,2nd & P,2nd & C,4th & P,4th & C,6th & P,6th \\
\hline RK3 & 0.3095 & 0.3078 & 0.4188 & 0.3583 & 0.6948 & 0.6624 \\
WJ & 0.3700 & 0.3698 & 0.4475 & 0.4186 & 0.6565 & 0.6358 \\
SOR & 0.0945 & 0.0442 & 0.1819 & 0.0339 & 0.4383 & 0.0495 \\
\hline \hline \hline$(\alpha, \beta)=(1,1 / 8)$ & C,2nd & P,2nd & C,4th & P,4th & C,6th & P,6th \\
\hline RK3 & 0.5079 & 0.5078 & 0.5720 & 0.5559 & 0.8006 & 0.7891 \\
WJ & 0.5893 & 0.5892 & 0.6303 & 0.6274 & 0.7804 & 0.7752 \\
SOR & 0.1171 & 0.0674 & 0.2230 & 0.0613 & 0.4675 & 0.0854 \\
\hline \hline
\end{tabular}

Table 2: Asymptotic convergence factors $\rho(M)$ with $\nu=5$ and $\alpha=1, \beta \in\{1 / 2,1 / 4,1 / 8\}$.

cretization of $(30)$ is

$$
\begin{aligned}
{\left[a\left(D_{1 x} \otimes I_{y}\right)+b\left(I_{x} \otimes D_{1 y}\right)\right] \mathbf{u}=\mathbf{f} } & +\varepsilon\left[\left(D_{2 x} \otimes I_{y}\right) \mathbf{u}+\left(I_{x} \otimes D_{2 y}\right)\right] \mathbf{u} \\
& -\left(P_{x}^{-1} E_{0 x} \otimes I_{y}\right)\left[\left(\left(a I_{x}-\varepsilon S_{x}\right) \otimes I_{y}\right) \mathbf{u}-\mathbf{g}_{W}\right] \\
& -\epsilon\left(I_{x} \otimes P_{y}^{-1} E_{N y}\right)\left[\left(I_{x} \otimes S_{y}\right) \mathbf{u}-\mathbf{g}_{N}\right] \\
& -\epsilon\left(P_{x}^{-1} E_{N x} \otimes I_{y}\right)\left[\left(S_{x} \otimes I_{y}\right) \mathbf{u}-\mathbf{g}_{E}\right] \\
& -\left(I_{x} \otimes P_{y}^{-1} E_{0 y}\right)\left[\left(I_{x} \otimes\left(b I_{y}-\varepsilon S_{y}\right)\right) \mathbf{u}-\mathbf{g}_{S}\right]
\end{aligned}
$$

and the corresponding problem augmented in dual time is strongly stable (see Appendix D). Thus, the smoothing techniques based on dual time marching are conditionally stable for (31).

The asymptotic convergence factors $\rho(M)$ for the multigrid algorithm applied to (30) with $a=b=1$ are displayed in Table 3 for RK3, WJ, SOR and $\varepsilon \in\{1,0.1,0.01\}$. Note that for $\varepsilon=1$, the results are comparable to the ones in Table 1.

Similarly to the purely elliptic problem (27), the multigrid convergence rates are improved by the SBP-preserving interpolation. The only exception is for the 4th order discretization with the weighted Jacobi smoothing technique and $\varepsilon=0.01$. Despite this fact, the convergence with the SBPpreserving grid transfer operators is faster even in this case, see Figure 7 . In 


\begin{tabular}{|c|c|c|c|c|c|c|c|c|}
\hline$\varepsilon=1$ & C,2nd & $\mathrm{P}, 2 \mathrm{nd}$ & C,4th & $\mathrm{P}, 4$ th & \multicolumn{4}{|c|}{ C,6th $\quad$ P,6th } \\
\hline RK3 & 0.1369 & 0.0761 & 0.2765 & 0.0879 & \multicolumn{2}{|c|}{0.5601} & 0.37 & \\
\hline WJ & 0.1542 & 0.0894 & 0.2624 & 0.1151 & \multicolumn{2}{|c|}{0.4938} & 0.33 & \\
\hline SOR & 0.0703 & 0.0232 & 0.1482 & 0.0133 & \multicolumn{2}{|c|}{0.4314} & 0.01 & \\
\hline$\varepsilon=0.1$ & C,2nd & $\mathrm{P}, 2 \mathrm{nd}$ & $\mathrm{C}, 4$ th & $\mathrm{P}, 4$ th & \multicolumn{2}{|c|}{ C,6th } & $\mathrm{P}, \mathrm{C}$ & \\
\hline RK3 & 0.1155 & 0.0725 & 0.2367 & 0.0848 & \multicolumn{2}{|c|}{0.4958} & 0.3 & 25 \\
\hline WJ & 0.1319 & 0.0859 & 0.2196 & 0.1068 & \multicolumn{2}{|c|}{0.4275} & 0.3 & 48 \\
\hline SOR & 0.0491 & 0.0187 & 0.0972 & 0.0128 & \multicolumn{2}{|c|}{0.3001} & 0.0 & 86 \\
\hline$\varepsilon=0.01$ & C,2nd & $\mathrm{P}, 2 \mathrm{nd}$ & C,4th & \multicolumn{2}{|c|}{$\overline{P \mathrm{P}, 4 \text { th }}$} & \multicolumn{2}{|c|}{ C,6th } & P,6th \\
\hline RK3 & 0.0603 & 0.0576 & 0.1032 & \multicolumn{2}{|c|}{0.1028} & \multicolumn{2}{|c|}{0.2095} & 0.1704 \\
\hline WJ & 0.0471 & 0.0448 & 0.0542 & \multicolumn{2}{|c|}{0.1073} & \multicolumn{2}{|c|}{0.2201} & 0.1840 \\
\hline SOR & $4.580 \mathrm{e}-6$ & $4.139 \mathrm{e}-6$ & $4.976 \mathrm{e}-5$ & \multicolumn{2}{|c|}{$2.528 \mathrm{e}-5$} & \multicolumn{2}{|c|}{0.0162} & 0.0078 \\
\hline
\end{tabular}

Table 3: Asymptotic convergence factors $\rho(M)$ with $\nu=5$ and $\epsilon \in\{1,0.1,0.01\}$.

fact, the SBP-preserving interpolation leads to faster (or comparable) convergence for all the cases reported in Table 3.

Remark 5.1. We stress that the spectral radius of the multigrid iteration matrix, $\rho(M)$, only indicates an asymptotic convergence behavior (see Remark 2.1). For small $\varepsilon$, the convergence rate of the multigrid method may have a plateau, as in Figure 7 , and hence the spectral radius might not always be a relevant measure of the speed of convergence.

\section{Summary and conclusions}

A new class of multigrid schemes for SBP-SAT discretizations has been proposed, where SBP-preserving interpolation operators have been used to transfer information between grids. When the Galerkin condition is considered, these prolongation and restriction operators lead to accurate and stable coarse-grid approximations.

Numerical experiments show that the SBP-preserving interpolation improves the convergence properties of the multigrid schemes for SBP-SAT discretizations, irrespective of the order of the discretization and the smoother chosen. Clear improvements are achieved for the Poisson problem, anisotropic elliptic equations and advection-diffusion problems. Moreover, the excellent 


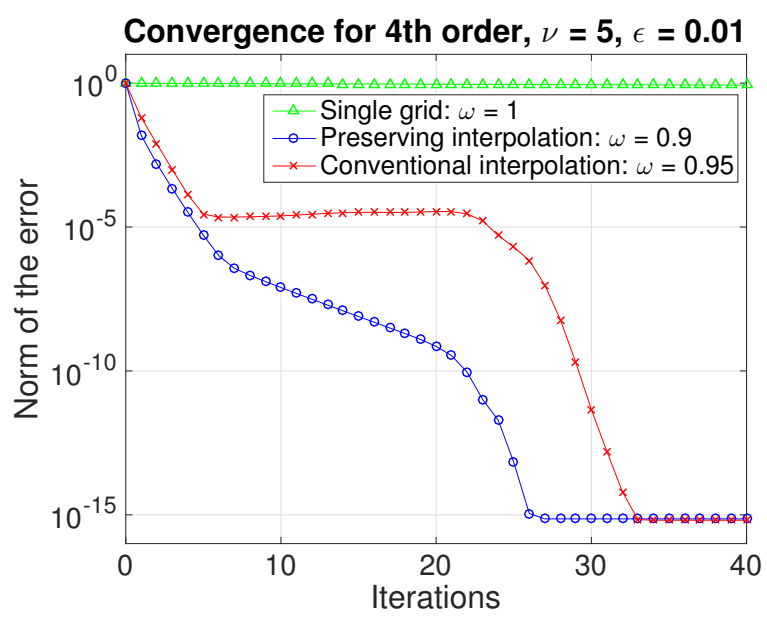

Figure 7: The convergence plot for a 4th-order accurate discretization with $\nu=5$ is shown for a weighted Jacobi smoother with optimal smoothing parameters. In particular, we considered the smoothing parameters $\omega=1$ (single-grid), 0.9 (SBP-preserving interpolation), 0.95 (conventional interpolation).

performance in combination with the smoother SOR, clearly indicate that multigrid algorithms with SBP-preserving interpolation can be designed to get very fast convergence.

In this paper we focused on steady model problems to compare the effect of different grid transfer operators. Our future efforts will be devoted to the study of multigrid techniques for time-dependent problems and systems of equations, for which an exact solve on coarse levels is not feasible.

\section{Acknowledgments}

We thank Dr. Tomas Lundquist for the useful suggestions regarding SBP-preserving interpolation and for providing the minimum bandwidth grid transfer operators that were used in the paper. This work was supported by VINNOVA, the Swedish Governmental Agency for Innovation Systems, under contract number 2013-01209.

\section{A. SBP-preserving interpolation operators}

The SBP-preserving interpolation operators for the second and fourth order of accuracy are taken from [31]. 


\section{A.1. Second order}

The discrete norm associated to the 2nd-order accurate interpolation operators is $P=\Delta x \operatorname{diag}\left(\frac{1}{2}, 1, \cdots, 1, \frac{1}{2}\right)$. The prolongation and restriction operators are given by

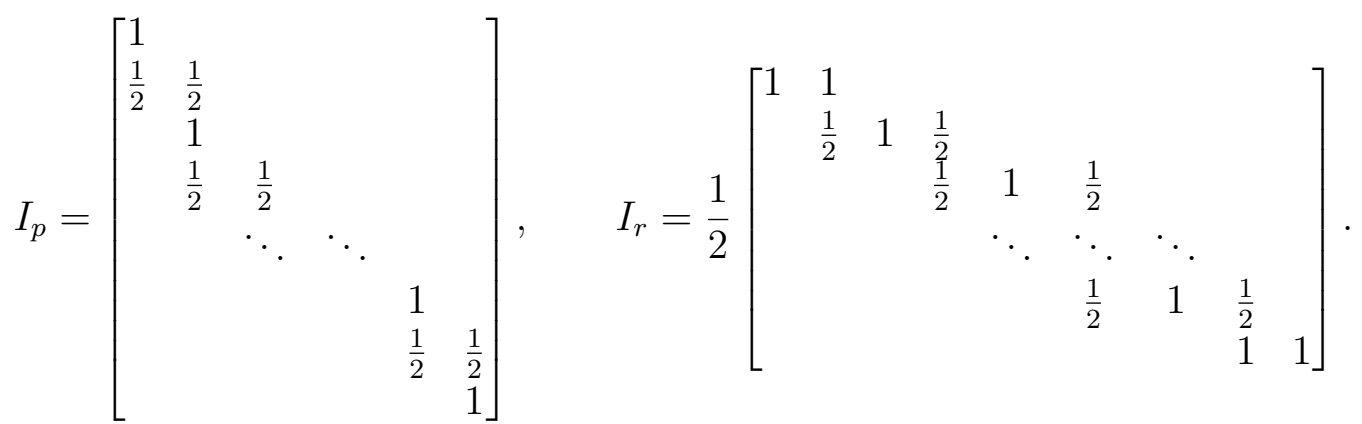

\section{A.2. Fourth order}

The discrete norm associated to the 4th-order accurate interpolation operators is $P=\Delta x \operatorname{diag}\left(\frac{17}{48}, \frac{59}{48}, \frac{43}{48}, \frac{49}{48}, 1, \ldots\right)$. The prolongation and restriction operators are given by

$$
\begin{aligned}
& I_{p}=\left[\begin{array}{cccccccccc}
1 & & & & & & & & \\
\frac{429}{944} & \frac{279}{472} & -\frac{43}{944} & & & & & & \\
& 1 & & & & & & & \\
-\frac{103}{784} & \frac{549}{784} & \frac{387}{784} & -\frac{1}{16} & & & & & \\
-\frac{5}{48} & \frac{5}{24} & \frac{43}{48} & & & & & & \\
-\frac{9}{256} & \frac{5}{256} & \frac{129}{256} & \frac{147}{256} & -\frac{1}{16} & & & & \\
\frac{1}{24} & -\frac{1}{16} & 0 & \frac{49}{48} & & & & & \\
\frac{23}{768} & -\frac{37}{768} & -\frac{43}{768} & \frac{147}{256} & \frac{9}{16} & -\frac{1}{16} & & & \\
-\frac{1}{384} & \frac{1}{256} & 0 & -\frac{49}{768} & \frac{9}{16} & \frac{9}{16} & -\frac{1}{16} & & \\
& & & & -\frac{1}{16} & \frac{9}{16} & \frac{9}{16} & -\frac{1}{16} & \\
& & & & & \ddots & \ddots & \ddots & \ddots
\end{array}\right], \\
& I_{r}=\left[\begin{array}{cccccccccc}
\frac{1}{2} & \frac{429}{544} & 0 & -\frac{103}{544} & -\frac{5}{34} & -\frac{27}{54} & \frac{1}{17} & \frac{23}{544} & 0 & -\frac{1}{272} \\
& \frac{279}{944} & \frac{43}{118} & \frac{549}{188} & \frac{5}{59} & \frac{15}{1888} & -\frac{3}{118} & -\frac{37}{1888} & 0 & \frac{3}{1888} \\
& -\frac{1}{32} & 0 & \frac{9}{32} & \frac{1}{2} & \frac{9}{32} & 0 & -\frac{1}{32} & & \\
& & \ddots & \ddots & \ddots & \ddots & \ddots & \ddots & \ddots &
\end{array}\right] \text {. }
\end{aligned}
$$




\section{A.3. Sixth order}

The discrete norm associated to the 6th-order accurate interpolation operators is $P=\Delta x \operatorname{diag}\left(\frac{13649}{43200}, \frac{12013}{8640}, \frac{2711}{4320}, \frac{5359}{4320}, \frac{7877}{8640}, \frac{43801}{43200}, 1, \ldots\right)$. The upper left corner of the restriction operator $I_{r}$ has as nonzero elements

$\begin{array}{ccc}a_{1,1}=0.5, & a_{1,2}=0.802123495768921, & a_{1,4}=-0.104402537502747, \\ a_{1,6}=-0.238706103698074, & a_{1,7}=-0.380613964392996, & a_{1,8}=0.035545186460547, \\ a_{1,9}=0.419261484357828, & a_{1,10}=0.205429427064253, & a_{1,11}=-0.132097589567002, \\ a_{1,12}=-0.122804755613961, & a_{1,14}=0.017813375750967, & a_{1,16}=-0.001548018627737 \\ a_{2,2}=0.390683205173562, & a_{2,3}=0.225672188462499, & a_{2,4}=0.234617900919837, \\ a_{2,6}=0.136350496597436, & a_{2,7}=0.259468908682261, & a_{2,8}=0.000056091577874, \\ a_{2,9}=-0.254058103720969, & a_{2,10}=-0.130230011237826, & a_{2,11}=0.075043702655457, \\ a_{2,12}=0.071821932489801, & a_{2,14}=-0.010305729990425, & a_{2,16}=0.000879418390493, \\ a_{3,2}=-0.173222951632239, & a_{3,4}=0.609132930652896, & a_{3,5}=0.726392475101439, \\ a_{3,6}=0.108261192825525, & a_{3,7}=-0.574880118037625, & a_{3,8}=-0.156462531123202, \\ a_{3,9}=0.422168941350055, & a_{3,10}=0.247894140769089, & a_{3,11}=-0.110844706750277 \\ a_{3,12}=-0.112912382423456, & a_{3,14}=0.015771970675023, & a_{3,16}=-0.001298961407228\end{array}$

while its interior stencil is $\left[a_{3}, 0, a_{2}, 0, a_{1}, a_{0}, a_{1}, 0, a_{2}, 0, a_{3}\right]$ with

$$
a_{0}=1 / 2, \quad a_{1}=75 / 256, \quad a_{2}=-25 / 512, \quad a_{3}=3 / 512 .
$$

\section{B. Proofs in section 4}

We start by discussing the claim of Proposition 4.3 and the accuracy properties of the coarse grid SBP-operator $D_{1,2}=I_{r} D_{1,1} I_{p}$. Here $D_{1,1}$ is the standard first derivative of order $(2 q, q)$ on the fine-grid and $I_{p}, I_{r}$ are $2 q$ th-accurate SBP-preserving interpolators.

\section{B.1. Proof of Proposition 4.3}

To prove that $D_{1,2}$ is an accurate representation of the first derivative on the coarse grid, we use $I_{p} \mathbf{x}_{2}^{k}=\mathbf{x}_{1}^{k}, I_{r} \mathbf{x}_{1}^{k}=\mathbf{x}_{2}^{k}, k=0, \ldots, q-1$ (cf. Definition 4.1), and the accuracy conditions $D_{1,1} \mathbf{x}_{1}^{k}=k \mathbf{x}_{1}^{k-1}, k=0, \ldots, q$. We find

$$
D_{1,2} \mathbf{x}_{2}^{k}=I_{r} D_{1,1} I_{p} \mathbf{x}_{2}^{k}=I_{r} D_{1,1} \mathbf{x}_{1}^{k}=k I_{r} \mathbf{x}_{1}^{k-1}=k \mathbf{x}_{2}^{k-1}, \quad k=0, \ldots, q-1,
$$

which proves that $D_{1,2}$ is at least a $(q-1)$ th-order accurate approximation of the first derivative on $\Omega_{2}$.

By reusing the previous argument, it is straightforward to verify that the accuracy conditions for $D_{1,2}$ on the interior nodes are satisfied for $k=$ $0, \ldots, 2 q-1$. To finalize the proof of Proposition 4.3 we must show that $D_{1,2}$ is $2 q$ th-order accurate in the interior. We will use the following 
Lemma B.1. Consider the interior stencil of a 2qth-order accurate prolongation operator

$$
\left(I_{p} \mathbf{f}\right)_{j}= \begin{cases}f_{i}, & j=2 i \\ \sum_{k=1}^{q} \alpha_{k}\left(f_{i+k}+f_{i+1-k}\right), & j=2 i+1,\end{cases}
$$

where the coefficients $\alpha_{k}$ are such that

$$
\begin{aligned}
\sum_{k=1}^{q} \alpha_{k} & =\frac{1}{2}, \\
\sum_{k=1}^{q} \alpha_{k}(2 k-1)^{2 m} & =0, \quad m=1, \ldots, q-1, \quad q>1 .
\end{aligned}
$$

The truncation error of the interpolation operator $I_{p}$ on the odd interior nodes is

$$
T_{e}((2 i+1) \Delta x)=\sum_{k=1}^{q} \frac{2 \alpha_{k}[(2 k-1) \Delta x]^{2 q}}{(2 q) !} f^{(2 q)}((2 i+1) \Delta x)+O\left(\Delta x^{2 q+1}\right) .
$$

Proof. The interpolation on the even nodes is exact, while the truncation error of the interpolation on the odd nodes can be estimated by Taylor expansion. For clarity, we show the result for $q=1$. The result for higher orders follows in a straightforward way.

The relation (B.1) on the odd nodes leads to

$$
\left(I_{p} \mathbf{f}\right)_{2 i+1}=\sum_{k=1}^{q} \alpha_{k}[f(2(i+k) \Delta x)+f(2(i+1-k) \Delta x)] .
$$

A Taylor expansion at $x=2 i+1$ gives

$$
\begin{aligned}
\left(I_{p} \mathbf{f}\right)_{2 i+1} & =f((2 i+1) \Delta x) \sum_{k=1}^{q} 2 \alpha_{k} \\
& +\sum_{k=1}^{q} \alpha_{k}[(2 k-1) \Delta x]^{2} f^{\prime \prime}((2 i+1) \Delta x)+O\left(\Delta x^{3}\right) .
\end{aligned}
$$

By using (B.2) we find

$$
\left(I_{p} \mathbf{f}\right)_{2 i+1}-f((2 i+1) \Delta x)=\sum_{k=1}^{q} \alpha_{k}[(2 k-1) \Delta x]^{2} f^{\prime \prime}((2 i+1) \Delta x)+O\left(\Delta x^{3}\right) .
$$

which proves (B.3) for $q=1$. 
Consider the function $f(x)=x^{2 q}$. By Lemma B.1, the truncation error of the prolongation on the interior nodes for this function has the structure $\mathbf{T}_{e}=c(\Delta x)^{2 q}[\ldots, 0,1,0,1,0, \ldots]^{T}$. This fine-grid function is canceled by all skew-symmetric stencils. In particular, the result of $D_{1,1} I_{p} \mathbf{x}_{2}^{2 q}$ is exact on the interior nodes, i.e. $D_{1,1} I_{p} \mathbf{x}_{2}^{2 q}=2 q \mathbf{x}_{1}^{2 q-1}$. The restriction operator $I_{r}$ operating on this vector does not generate error, since $I_{r}$ exactly restricts $(2 q-1)$ th-order polynomials on the interior nodes. Therefore, the order of accuracy of the first derivative operator on the coarse grid is $(2 q, q-1)$ and Proposition 4.3 follows.

\section{B.2. Proof of Proposition 4.8}

Next, we discuss Proposition 4.8. It is straightforward to verify that $D_{2,2}=I_{r} D_{2,1} I_{p}$ exactly mimics the second derivative of $x^{k}$ for $k=0, \ldots, 2 q-$ 1 in the interior nodes and for $k=0, \ldots, q-1$ at the boundaries. To prove that the accuracy of $D_{2,2}$ in the interior nodes is the same as the one of $D_{2,1}$, we use Lemma B. 1 for $k \in\{2 q, 2 q+1\}$.

Remark B.2. Since we are only interested to the behavior of $D_{2,2}$ in the interior, we consider an infinite grid with equidistant nodes.

Let $f(x)=x^{2 q}$. From Lemma B.1, the truncation error of $I_{p} \mathbf{f}$ has the structure $\mathbf{T}_{e}=c(\Delta x)^{2 q}[\ldots, 0,1,0,1,0, \ldots]^{T}$. Thus, by applying the finegrid operator we find $D_{2,1} \mathbf{T}_{e}=c(\Delta x)^{2 q}\left[\ldots, s_{e}, s_{o}, s_{e}, s_{o}, \ldots\right]^{T}$, where $s_{e}, s_{o}$ are the sums of the elements of $D_{2,1}$ on even (e) and odd (o) column positions, respectively. Since $D_{2,1}$ is at least 0th-order accurate $s_{o}=-s_{e}$, and $D_{2,1} \mathbf{T}_{e}=c s_{e}(\Delta x)^{2 q}[\ldots, 1,-1,1,-1, \ldots]^{T}$. This vector is in the nullspace of any consistent restriction operator such that (cf. Appendix A and (B.1))

$$
\left(I_{r} \mathbf{v}\right)_{j}=\frac{1}{2}\left[v_{2 j}+\sum_{k=1}^{q} \alpha_{k}\left(v_{2 j-1+2 k}+v_{2 j+1-2 k}\right)\right]
$$

and (B.2) hold. Hence $D_{2,2}$ is exact on $2 q$ th-order polynomials.

Next, consider $f(x)=x^{2 q+1}$. Lemma B.1 implies that the truncation error of $I_{p} \mathbf{f}$ has the structure $\mathbf{T}_{e}=c(\Delta x)^{2 q+1}[\ldots, 0,2 i+1,0,2 i+3,0, \ldots]^{T}$. 
This vector can be decomposed as

$$
\begin{aligned}
\mathbf{T}_{e} & =c(\Delta x)^{2 q+1}\left[\ldots, i, \frac{2 i+1}{2}, i+1, \frac{2 i+3}{2}, i+2, \ldots\right]^{T} \\
& +c(\Delta x)^{2 q+1}\left[\ldots,-i, \frac{2 i+1}{2},-(i+1), \frac{2 i+3}{2},-(i+2), \ldots\right]^{T} \\
& =\mathbf{T}_{n}+\mathbf{T}_{p},
\end{aligned}
$$

where $\mathbf{T}_{n}$ is proportional to $\mathbf{x}_{1}$ and hence vanishes when $D_{2,1}$ is applied to it for $q \geq 1$. The vector $\mathbf{T}_{p}$ can be written element-wise as $c(\Delta x)^{2 q+1}(-1)^{j} j$. Since $D_{2,1}$ has a symmetric stencil, i.e.

$$
\left(D_{2,1} \mathbf{v}\right)_{j}=c_{0} v_{j}+\sum_{k=1}^{q} c_{j}\left(v_{j+k}+v_{j-k}\right)
$$

we find $\left(D_{2,1} \mathbf{T}_{p}\right)_{j}=(-1)^{j+1} j\left(-c_{0}+2 \sum_{k=1}^{q} c_{k}\right)=C(-1)^{j} j$. Finally, by applying $I_{r}$ in (B.4) to this vector and using the accuracy requirement (B.2), we find

$$
\left(I_{r} D_{2,1} \mathbf{T}_{p}\right)_{j}=2 j\left(1-2 \sum_{k=1}^{q} \alpha_{k}\right)=0 .
$$

Thus, $D_{2,2}$ is exact on $(2 q+1)$ th-order polynomials, as well.

\section{Energy estimates for the elliptic problem}

Consider the problem (27) augmented in dual time, i.e.

$$
\begin{aligned}
u_{\tau}-\alpha u_{x x}-\beta u_{y y} & =f, & & 0<x<1, \quad 0<y<1, \quad \tau>0, \\
\left(u-u_{x}\right)(0, y, \tau) & =g_{W}(y), & & 0<y<1, \tau>0, \\
\left(u+u_{y}\right)(x, 1, \tau) & =g_{N}(x), & & 0<x<1, \quad \tau>0, \\
\left(u+u_{x}\right)(1, y, \tau) & =g_{E}(y), & & 0<y<1, \quad \tau>0 \\
\left(u-u_{y}\right)(x, 0, \tau) & =g_{S}(x), & & 0<x<1, \quad \tau>0 \\
u(x, y, 0) & =u^{(0)}(x, y), & & 0<x<1, \quad 0<y<1 .
\end{aligned}
$$

By using the energy-method (multiplying (C.1) by $u$, integrating on the square domain $\Omega=(0,1)^{2}$ and applying integration-by-parts) we find

$$
\begin{aligned}
\frac{\mathrm{d}}{\mathrm{d} \tau}\|u(\cdot, \tau)\|_{2}^{2} & =2 \alpha \int_{0}^{1}\left[u u_{x}(1, y, \tau)-u u_{x}(0, y, \tau)\right] \mathrm{d} y \\
& +2 \beta \int_{0}^{1}\left[u u_{y}(x, 1, \tau)-u u_{y}(x, 0, \tau)\right] \mathrm{d} x \\
& -2 \int_{\Omega}\left(\alpha u_{x}^{2}+\beta u_{y}^{2}\right) \mathrm{d} x \mathrm{~d} y+2(f, u)_{2},
\end{aligned}
$$


where $(\cdot, \cdot)_{2}$ and $\|\cdot\|_{2}$ denote the scalar product and the norm on $L^{2}(\Omega)$, respectively. To start with, consider the case with $f=0$. Since both $\alpha$ and $\beta$ are positive constants, the only terms that can lead to a positive energyrate are related to the boundaries. In particular

$$
2 u u_{x}=\frac{1}{2}\left(u+u_{x}\right)^{2}-\frac{1}{2}\left(u-u_{x}\right)^{2}, \quad 2 u u_{y}=\frac{1}{2}\left(u+u_{y}\right)^{2}-\frac{1}{2}\left(u-u_{y}\right)^{2}
$$

and the strong imposition of the boundary conditions of (C.1) leads to an energy estimate, since all the positive contributions to the energy-rate are controlled by the data. If $f \neq 0$, the energy-estimate includes a limited exponential growth.

Likewise, the proof of strong stability of (29) relies on the discrete energymethod (multiplying the formulation from the left by $\mathbf{w}^{T}\left(P_{x} \otimes P_{y}\right)$ and applying the summation-by-parts properties), which leads to

$$
\begin{aligned}
\frac{\mathrm{d}}{\mathrm{d} \tau}\|\mathbf{w}(\tau)\|_{P_{x} \otimes P_{y}}^{2} & =-2 \alpha \mathbf{w}^{T}\left(S_{x}^{T} A_{x} S_{x} \otimes P_{y}\right) \mathbf{w}-2 \beta \mathbf{w}^{T}\left(P_{x} \otimes S_{y}^{T} A_{y} S_{y}\right) \mathbf{w} \\
& -2 \alpha \mathbf{w}^{T}\left(E_{0 x} \otimes P_{y}\right)\left(\mathbf{w}-\mathbf{g}_{W}\right)-2 \beta \mathbf{w}^{T}\left(P_{x} \otimes E_{N y}\right)\left(\mathbf{w}-\mathbf{g}_{N}\right) \\
& -2 \alpha \mathbf{w}^{T}\left(E_{N x} \otimes P_{y}\right)\left(\mathbf{w}-\mathbf{g}_{E}\right)-2 \beta \mathbf{w}^{T}\left(P_{x} \otimes E_{0 y}\right)\left(\mathbf{w}-\mathbf{g}_{S}\right) \\
& +2(\mathbf{w}, \mathbf{f})_{P_{x} \otimes P_{y}} .
\end{aligned}
$$

Similarly to the continuous case, the boundedness of the discrete energy-rate depends only on the boundary terms, since $A_{x}+A_{x} \geq 0$ and $A_{y}+A_{y}^{T} \geq 0$. As an example, the western-boundary terms can be rewritten as

$$
\begin{aligned}
-2 \alpha \mathbf{w}^{T}\left(E_{0 x} \otimes P_{y}\right)\left(\mathbf{w}-\mathbf{g}_{W}\right)= & -2 \alpha\left(\mathbf{w}-\mathbf{g}_{W}\right)^{T}\left(E_{0 x} \otimes P_{y}\right)\left(\mathbf{w}-\mathbf{g}_{W}\right) \\
& +\frac{1}{2} \alpha \mathbf{g}_{W}^{T}\left(E_{0 x} \otimes P_{x}\right) \mathbf{g}_{W}
\end{aligned}
$$

which yields a bounded contribution. The remaining boundary terms can be recast in the same fashion, leading to a discrete energy-estimate. 


\section{Energy estimates for the advection-diffusion problem}

Consider the problem (30) augmented in dual time, i.e.

$$
\begin{aligned}
u_{\tau}+a u_{x}+b u_{y} & =\varepsilon\left(u_{x x}+u_{y y}\right)+f, & 0<x<1, & 0<y<1, \quad \tau>0, \\
\left(a u-\varepsilon u_{x}\right)(0, y, \tau) & =g_{W}(y), & & 0<y<1, \quad \tau>0, \\
u_{y}(x, 1, \tau) & =g_{N}(x), & & 0<x<1, \quad \tau>0, \\
u_{x}(1, y, \tau) & =g_{E}(y), & & 0<y<1, \quad \tau>0, \\
\left(b u-\varepsilon u_{y}\right)(x, 0, \tau) & =g_{S}(x), & & 0<x<1, \quad \tau>0, \\
u(x, y, 0) & =u^{(0)}(x, y), & & 0<x<1, \quad 0<y<1,
\end{aligned}
$$

and the corresponding semi-discrete formulation

$$
\begin{aligned}
\mathbf{w}_{\tau}+\left[a\left(D_{1 x} \otimes I_{y}\right)+b\left(I_{x} \otimes\right.\right. & \left.\left.D_{1 y}\right)\right] \mathbf{w}=\mathbf{f}+\varepsilon\left[\left(D_{2 x} \otimes I_{y}\right) \mathbf{w}+\left(I_{x} \otimes D_{2 y}\right)\right] \mathbf{w} \\
& -\left(P_{x}^{-1} E_{0 x} \otimes I_{y}\right)\left[\left(\left(a I_{x}-\varepsilon S_{x}\right) \otimes I_{y}\right) \mathbf{w}-\mathbf{g}_{W}\right] \\
& -\epsilon\left(I_{x} \otimes P_{y}^{-1} E_{N y}\right)\left[\left(I_{x} \otimes S_{y}\right) \mathbf{w}-\mathbf{g}_{N}\right] \\
& -\epsilon\left(P_{x}^{-1} E_{N x} \otimes I_{y}\right)\left[\left(S_{x} \otimes I_{y}\right) \mathbf{w}-\mathbf{g}_{E}\right] \\
& -\left(I_{x} \otimes P_{y}^{-1} E_{0 y}\right)\left[\left(I_{x} \otimes\left(b I_{y}-\varepsilon S_{y}\right)\right) \mathbf{w}-\mathbf{g}_{S}\right]
\end{aligned}
$$

The proof of well-posedness follows from the energy-method, which leads to

$$
\begin{aligned}
\frac{\mathrm{d}}{\mathrm{d} \tau}\|u(\cdot, \tau)\|_{2}^{2} & =\left.\int_{0}^{1}\left[2 \varepsilon u u_{x}-a u^{2}\right]\right|_{x=0} ^{x=1} \mathrm{~d} y+\left.\int_{0}^{1}\left[2 \varepsilon u u_{y}-b u^{2}\right]\right|_{y=0} ^{y=1} \mathrm{~d} x \\
& -2 \varepsilon \int_{\Omega}\left(u_{x}^{2}+u_{y}^{2}\right) \mathrm{d} x \mathrm{~d} y+2(f, u)_{2} .
\end{aligned}
$$

Since

$$
\begin{aligned}
2 \varepsilon u u_{x}-a u^{2} & =\frac{1}{a}\left[a^{2} \varepsilon^{2} u_{x}^{2}-\left(a u-\varepsilon u_{x}\right)^{2}\right], \\
2 \varepsilon u u_{y}-b u^{2} & =\frac{1}{b}\left[b^{2} \varepsilon^{2} u_{y}^{2}-\left(b u-\varepsilon u_{y}\right)^{2}\right],
\end{aligned}
$$

the boundary conditions in (D.1) yield a solution bounded by the data.

To prove strong stability of (D.2) we apply the discrete energy-method 
as in Appendix $\mathrm{C}$, which gives

$$
\begin{aligned}
\frac{\mathrm{d}}{\mathrm{d} \tau}\|\mathbf{w}(\tau)\|_{P_{x} \otimes P_{y}}^{2}= & -2 \varepsilon \mathbf{w}^{T}\left[\left(S_{x}^{T} A_{x} S_{x} \otimes P_{y}\right)+\left(P_{x} \otimes S_{y}^{T} A_{y} S_{y}\right)\right] \mathbf{w} \\
& +a \mathbf{w}^{T}\left(E_{0 x} \otimes P_{y}\right) \mathbf{w}-2 \mathbf{w}^{T}\left(E_{0 x} \otimes P_{y}\right)\left(a \mathbf{w}-\mathbf{g}_{W}\right) \\
& -b \mathbf{w}^{T}\left(P_{x} \otimes E_{N y}\right) \mathbf{w}+2 \varepsilon \mathbf{w}^{T}\left(P_{x} \otimes E_{N y}\right) \mathbf{g}_{N} \\
& -a \mathbf{w}^{T}\left(E_{N x} \otimes P_{y}\right) \mathbf{w}+2 \varepsilon \mathbf{w}^{T}\left(E_{N x} \otimes P_{y}\right) \mathbf{g}_{E} \\
& +b \mathbf{w}^{T}\left(P_{x} \otimes E_{0 y}\right) \mathbf{w}-2 \mathbf{w}^{T}\left(P_{x} \otimes E_{0 y}\right)\left(b \mathbf{w}-\mathbf{g}_{S}\right) \\
& +2(\mathbf{w}, \mathbf{f})_{P_{x} \otimes P_{y}}
\end{aligned}
$$

The western-boundary terms are equivalent to

$$
-\frac{1}{a}\left(a \mathbf{w}-\mathbf{g}_{W}\right)^{T}\left(E_{0 x} \otimes P_{y}\right)\left(a \mathbf{w}-\mathbf{g}_{W}\right)+\frac{1}{a} \mathbf{g}_{W}^{T}\left(E_{0 x} \otimes P_{y}\right) \mathbf{g}_{W},
$$

and the northern-boundary terms can be recast as follows

$$
-\frac{1}{b}\left(b \mathbf{w}-\varepsilon \mathbf{g}_{N}\right)^{T}\left(P_{x} \otimes E_{N y}\right)\left(b \mathbf{w}-\varepsilon \mathbf{g}_{N}\right)+\frac{\varepsilon^{2}}{b} \mathbf{g}_{N}^{T}\left(P_{x} \otimes E_{N y}\right) \mathbf{g}_{N} .
$$

The eastern- and southern-boundary terms can be treated in a similar manner. Thus, (D.3) leads to a discrete energy-estimate.

\section{References}

[1] R. P. Fedorenko, Iterative Methods for Elliptic Difference Equations, Russian Mathematical Surveys, Vol. 28, pp. 129-195, 1973.

[2] U. Trottenberg, C.W. Oosterlee, A. Schuller, Multigrid, Academic Press, 2000 .

[3] W. L. Briggs, V. E. Henson, S. F. McCormick, A Multigrid Tutorial, Second Edition, SIAM, 2000.

[4] A. Brandt, N. Dinar, Multigrid Solutions to Elliptic Flow Problems, in: S. V. Parter, Numerical Methods for Partial Differential Equations, Academic Press, 1979.

[5] E. Katzer, Multigrid Methods for Hyperbolic Equations, in Multigrid Methods III, International Series of Numerical Mathematics, Vol. 98, pp 253-263, 1991. 
[6] C. Stolk, M. Ahmed, S. K. Bhowmik, A Multigrid Method for the Helmholtz Equation with Optimized Coarse Grid Corrections, SIAM Journal on Scientific Computing, Volume 36, Issue 6, 2014.

[7] J. H. Adler et al., Monolithic Multigrid Methods for Two-Dimensional Resistive Magnetohydrodynamics, SIAM Journal on Scientific Computing, Volume 38, pp. B1-B24, 2016.

[8] C. D. Santiago, C. H. Marchi, L. F. Souza, Performance of geometric multigrid method for coupled two-dimensional systems in CFD, Applied Mathematical Modelling, Volume 39, Issue 9, pp. 2602-2616, 2015.

[9] K. Mattson, M. H. Carpenter, Stable and Accurate Interpolation Operators for High-Order Multi-Block Finite-Difference Methods, SIAM Journal on Scientific Computing, Volume 32, Issue 4, 2010.

[10] M. Svärd, J. Nordström, Review of Summation-By-Parts Schemes for Initial-Boundary-Value Problems, Journal of Computational Physics, Volume 268, pp. 17-38, 2014.

[11] K. Mattsson, M. Svärd, J. Nordström, Stable and Accurate Artificial Dissipation, Journal of Scientific Computing, Vol. 21, pp.57-79, 2004.

[12] B. Gustafsson, H. O. Kreiss, J. Oliger, Time Dependent Problems and Difference Methods, John Wiley \& Sons, Inc., 1995.

[13] J. Nordström, A Roadmap to Well Posed and Stable Problems in Computational Physics, Journal of Scientific Computing, Vol. 71, pp. 365-385, 2017.

[14] S. McCormick, Multigrid methods, SIAM, 1987.

[15] W. Hackbusch, Multi-Grid Methods and Applications, Springer, 1985.

[16] L. Hogben, Handbook of Linear Algebra, CRC Press, p. 2-12, 2007.

[17] B. Strand, Summation by Parts for Finite Difference Approximations for $d / d x$, Journal of Computational Physics, Volume 110, pp. 47-67, 1994. 
[18] D. C. Del Rey, P. D. Boom, D. W. Zingg, A generalized framework for nodal first derivative summation-by-parts operators, Journal of Computational Physics, Vol. 266, pp. 214-239, 2014.

[19] M. H. Carpenter, D. Gottlieb, Spectral Methods on Arbitrary Grids, Journal of Computational Physics, Vol. 129, pp. 74-86, 1996.

[20] J. Nordström, Conservative Finite Difference Formulations, Variable Coefficients, Energy Estimates and Artificial Dissipation, Journal of Scientific Computing, Vol. 29, pp.375-404, 2006.

[21] T. C. Fisher, M. H. Carpenter, J. Nordström, N. K. Yamaleev, C. Swanson, Discretely conservative finite-difference formulations for nonlinear conservation laws in split form: Theory and boundary conditions, Journal of Computational Physics, Vol. 234, pp. 353-375, 2013.

[22] M. Svärd, On coordinate transformation for summation-by-parts operators, Journal of Scientific Computing, Vol. 20, Issue 1, 2004.

[23] M. H. Carpenter, J. Nordström, D. Gottlieb, A stable and conservative interface treatment of arbitrary spatial accuracy, Journal of Computational Physics, Vol. 148, pp. 341-365, 1999.

[24] K. Mattsson, J. Nordström, Summation by parts operators for finite difference approximations of second derivatives, Journal of Computational Physics, Vol. 199, pp. 503-540, 2004.

[25] M. H. Carpenter, D. Gottlieb, S. Abarbanel, Time-stable boundary conditions for finite-difference schemes solving hyperbolic systems: Methodology and application to high-order compact schemes, Journal of Computational Physics, Volume 111 No. 2, pp. 220-236, 1994.

[26] D. C. Del Rey, J. E. Hicken, D. W. Zingg, Review of summation-byparts operators with simultaneous approximation terms for the numerical solution of partial differential equations, Computers \& Fluids, Vol. 95, pp. 171-196, 2014.

[27] P. W. Hemker, On the order of prolongations and restrictions in multigrid procedures, Journal of Computational and Applied Mathematics, Vol. 32, pp. 423-429, 1990. 
[28] H. Sundar, G. Stadler, G. Biros, Comparison of Multigrid Algorithms for High-Order Continuous Finite Element Discretizations, Numerical Linear Algebra with Applications, Vol. 22, pp. 664-680, 2015.

[29] Y. Saad, Iterative Methods for Sparse Linear Systems, Second Edition, SIAM, 2003.

[30] W. Hackbusch, The Frequency Decomposition Multi-Grid Method, Numerische Matematik, Vol. 56, pp. 229-245, 1989.

[31] T. Lundquist, J. Nordström, On the Suboptimal Accuracy of Summationby-parts Schemes with Non-conforming Block Interfaces, Technical report, LiTH-MAT-R-2015/16-SE, 2015. 